\title{
How to create a full-wave GPR model of a 3D domain of railway track bed?
}

\author{
M.G. Brancadoro \\ Department of Engineering, Roma Tre University, Rome, Italy
}

F. Tosti

School of Computing and Engineering, University of West London, London, United Kingdom

\author{
L. Bianchini Ciampoli \\ Department of Engineering, Roma Tre University, Rome, Italy
}

\section{Pajewski}

Department of Information Engineering, Electronics and Telecommunications, Sapienza University of Rome, Italy

\author{
D. Pirrone \& A. Benedetto \\ Department of Engineering, Roma Tre University, Rome, Italy
}

\author{
A.M. Alani \\ School of Computing and Engineering, University of West London, London, United Kingdom
}

\begin{abstract}
Ground-penetrating radar (GPR) investigations of railway track beds are becoming more important nowadays in civil engineering. The manufacturing of representative full-scale scenarios in the laboratory environment for the creation of databases can be a critical issue. It is difficult to reproduce and monitor the effect of differing physical and performance parameters in the ballast layer as well as to evaluate the combination of these factors in more complex scenarios. In addition, reproducing full-scale tests of railway ballast implies to handle huge amounts of aggregates. To this effect, the use of the Finite-Difference TimeDomain (FDTD) simulation of the ground-penetrating radar signal can represent a powerful tool for creating, extending or validating databases difficult to build up and to monitor at the real scale of investigation. Nevertheless, a realistic three-dimensional simulation of a railway structure requires huge computational efforts. This work focuses on performing simulation of the ground-penetrating radar signal within a railway track bed by using a two-dimensional cross-section model of the ballast layer, generated by a Random Sequential Adsorption (RSA) paradigm. Attention was paid on the geometric reconstruction of the ballast system as well as on the content of voids between the aggregate particles, which complied with the real-world conditions of compaction for this material. The resulting synthetic GPR signal was subsequently compared with the real signal collected within a realistic track bed scenario of ballast aggregates recreated in the laboratory environment.
\end{abstract}

\section{INTRODUCTION}

The effective monitoring of ballast conditions has a crucial role in railway engineering for ensuring proper operation of the rail trains and safety of passengers. Rail ballast is usually made of coarse aggregates derived by crushed rocks and characterized by a quasi-uniform grading. Its main functions are to maintain the arrangement of the rail tracks and to ensure the structural capacity of the track bed (Selig \& Waters 1974, Indraratna 2016).

Ground-penetrating radar (GPR) is being increasingly used in railway applications as a powerful nondestructive technique for the efficient monitoring of the conditions of railway track beds (Hugenschmidt 2000). Many research activities have focused on the assessment of ballast conditions over the last years, in both laboratory (Roberts et al. 2006, Al-Qadi et al. 2008, 2010, De Chiara et al. 2014) and real-world environment (Hugenschmidt 2000, Olhoeft \& Selig 2002, Roberts et al. 2007, Benedetto \& Pajewski 2015). The main drawbacks of the application of GPR in this area concerns the difficulties in reproducing and controlling the effect of differing physical and performance parameters in the ballast layer as well as to evaluate the combination of these factors in more complex scenarios. In addition, reproducing full-scale tests of railway ballast is timeconsuming and it implies to handle huge amounts of aggregates. Thereby, the creation of real-scale track bed scenarios in a numerical simulation environment can represent a powerful tool for the investigation of the railway ballast conditions, as it allows to perform rapid and multiple tests. Furthermore, the simulation 
enables to analyse, in a controlled environment, differing conditions that can be hardly reproduced in the laboratory, as well as to generate a large amount of ballast samples with differing physical conditions.

On the other hand, it is relatively complex to simulate a GPR signal for a railway track bed. Electromagnetically speaking, it is necessary to calibrate the physical properties of the investigated materials and to create samples with representative threedimensional (3D) characteristics in order to ensure consistency between the real and the simulated sample.

The reproduction of such an irregular volumetric ensemble of coarse aggregates represents a nonnegligible numerical problem, which necessarily requires simplifications to limit the computational efforts.

By literature, the most acknowledged method for the simulation of railway ballast is based on the representation of the polyhedral-shaped aggregates using a cluster of smaller simple shapes (Thakur et al. 2009, Indraratna et al. 2016, Sharif et al. 2016). In practical terms, this "clump logic" method allows to simulate the bi-dimensional (2D) or 3D irregular geometry of the ballast grains through the connection and overlapping of a number of smaller spheres (i.e., 3D domain), or circles (i.e., 2D domain), all of which are characterized by differing sizes and positions.

In this study, a novel methodology for the simulation of railway ballast is proposed. The method is based on the Random Sequential Adsorption (RSA) paradigm (Feder 1980), which ensures the random location of randomly-sized ballast particles within a simulation domain consistent with the actual dimensions of ballast layers in the real-life environment. The size of the ballast grains was generated according to the grading of the aggregates used in the realcase test. The GPR signal of the produced scenario was subsequently obtained using the FiniteDifference Time-Domain (FDTD) paradigm for the generation of synthetic GPR signals.

\section{METHODOLOGY}

The proposed method is based on the $2 \mathrm{D}$ simulation of a GPR signal emitted towards a railway track bed scenario. To this effect, two combined numerical paradigms have been used. The first one aims at reproducing a virtual scenario of ballast particles with a bi-dimensional cross-section model of a real railway track bed. The second paradigm allows for the generation of the relevant synthetic GPR signal from such a virtual scenario. The two algorithms are run together in the gprMax numerical simulator (Giannopoulos 2005). The freeware tool $E^{2} \mathrm{GPR}$ aided the design of gprMax models and their distributed execution on multicore machines (Pirrone \& Pajewski 2015).

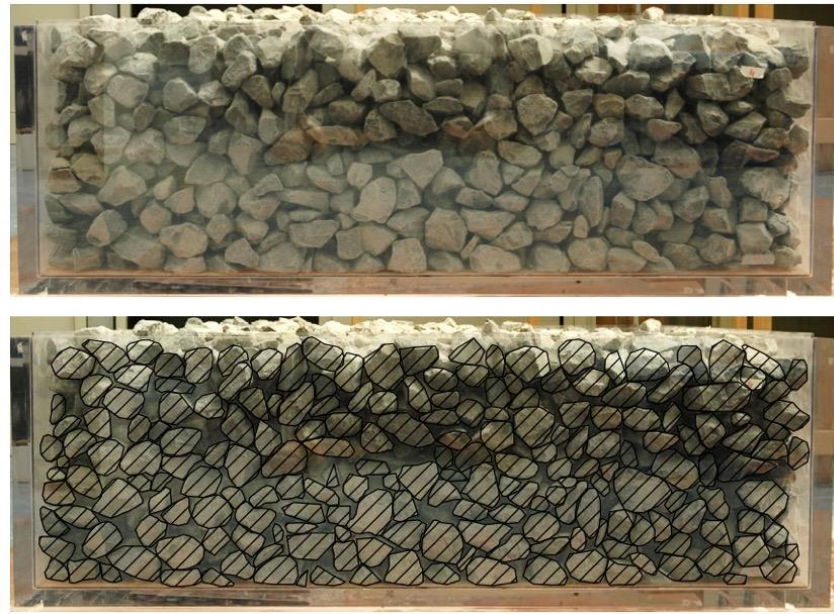

Figure 1. - Side-view of the laboratory set-up composed of a methacrylate tank filled-up with railway ballast aggregates (above) and the contour lines of the aggregates on the ideal cross-sectional plan visible from the side wall (below).

The proposed numerical simulation methodology is based on two main simplifications on both the simulation domain and the ballast particles. As mentioned above, the simulation of a 2D domain was performed to lower the computational efforts required for both the generation of the ballast sample domain and the simulation of the synthetic EM signals. In addition, the ballast particles have been schematically represented by an ensemble of circumferences with differing diameters. Although this geometry is not representative of the highly irregular shape of real ballast aggregates, it well stands for the uniform grading and the high voids content that typically characterize the railway ballast. Furthermore, such a simple geometry allows to run a completely random algorithm, as explained in Section 2.2. To validate the methodology, the retrieved synthetic signal was compared to the real GPR signal collected in the laboratory environment.

\subsection{From $3 D$ to $2 D$}

First steps for representing the 3D volume of a railway track bed in a 2D plan are to recreate the track bed cross section, and to assign the relevant dielectric properties to each embedded material. In Figure 1 , it is shown the laboratory set-up reproducing the ballast layer of a railway track bed. The methacrylate framework allows a clear side-view, which is actually a cross-section of the domain.

The main issue with such an approach is that the $2 \mathrm{D}$ ratio between the air and the solid components is slightly lower than in the 3D case. As an example, let us consider a sphere in a cube with side dimension equal to the sphere diameter, and a circle in a square with the same dimension. The ratio between the air- and the solid-filled volumes or areas, depending on the case, is $\pi / 6$ and $\pi / 4$, respectively. As a result, the $3 \mathrm{D}$ voids ratio is $33 \%$ higher than the 2D for this simplified model. If we consider the dielectric permittivity of the whole system, this fact 
turns into a simulation domain which is not representative of the real case, as the relative dielectric permittivity of the air-ballast mix is highly dependent on the content of voids.

To this effect, the following condition must be respected when attempting to represent a 3D domain of a railway track bed using a $2 \mathrm{D}$ cross-sectional model:

$\frac{V_{v}}{V_{b}}=\frac{A_{v}}{A_{b}}=K$

with the characters $V, A, v$ and $b$ standing for 'volume', 'area', 'voids' and 'ballast', respectively, and $K$ being the voids index of the ballast-air mix. In view of this, the simulated $2 \mathrm{D}$ domain was made representative of the real 3D volumetric configuration (i.e., using the air/ballast grain volumetric ratio of Equation 1), rather than of the air/ballast grain surface ratio on the cross-sectional plan of the $3 \mathrm{D}$ case (i.e., considering the contour lines of the aggregates in Figure 1).

\subsection{Location of the ballast particles by the RSA paradigm}

The simulation paradigm used for reproducing the solid matrix of the railway ballast is inspired by the RSA paradigm (Feder 1980). The RSA is an irreversible model, utilised in many scientific fields (Lee 2000, Talbot et al. 2000) to randomly fill up a $d$-dimensional space with multiple objects, under a non-overlapping condition.

According to the RSA algorithm, when reproducing a $2 \mathrm{D}$ railway ballast sample, a random sequence is adopted to define the dimension of the ballast particles and their positions within the simulation domain. Under the assumption of circle-shaped ballast aggregates, a set of diameters (complying with the actual grading of the material under simulation) is sequentially and randomly extracted. Subsequently, the position of each circle is randomly determined complying with the twofold condition that the new

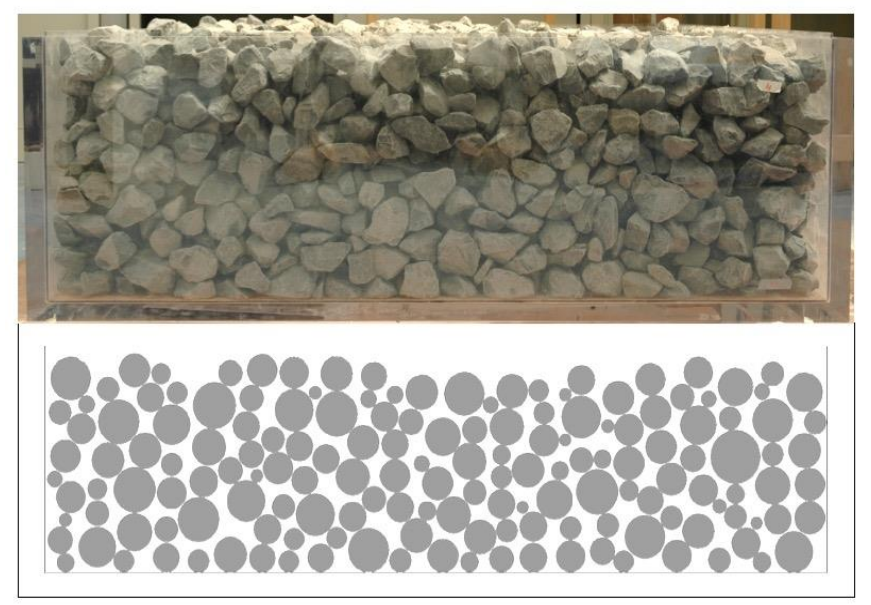

Figure 2. - Laboratory set-up composed of a methacrylate framework filled-up with railway ballast (above) and corresponding 2D simulation by the RSA approach (below). particle completely belongs to the simulation domain, and it does not overlap with any other.

Finally, the last conditions of the RSA paradigm ensure the contacts between the aggregates as per the real conditions of the ballast layer within a track bed. Thereby, a 'gravitational' automatic compaction process is performed, where the particle are shifted downwards along the vertical direction, up to the contact points with the grains below. The rationale of this process is to reproduce gravitational effects during the laying phase of the material (Figure 2). Such a simulation paradigm requires two input parameters, namely, the grain size distribution and the compaction rate of the ballast, as they can both influence the voids index of the air-ballast mix. Due to the effects of the air voids on the dielectric behaviour of the material, the correct definition of these parameters becomes crucial to ensure that the electromagnetic (EM) response of the 2D domain is consistent with that obtained by the real-case GPR test, as mentioned in Section 2.1. In practical terms, the value of the real-case voids index $K$ (see Equation 1) must be an imposed condition to the simulation domain, in order to subsequently define the area to be filled by the ballast particles.

\section{METHOD APPLICATION}

The GPR data collected in the laboratory environment were compared with those obtained by using sequentially the RSA and the FDTD paradigms.

In more detail, real GPR tests have been conducted on a set-up composed of a $1.55 \mathrm{~m}$ wide $\times 1.55 \mathrm{~m}$ long $\times 0.50 \mathrm{~m}$ high methacrylate tank laid onto a metal plate and filled up with limestone-derived railway ballast. Static GPR data have been collected using a horn antenna, with a central frequency of $1000 \mathrm{MHz}$, which was set to work suspended in the air at $0.40 \mathrm{~m}$ height from the ballast surface (Figure 3). More details about the set-up can be found in (Tosti et al. 2016). The geometric and the EM characterization of the material composing the test domain are summarised in Table 1.

Table 1. Main geometric and dielectric properties of the tested ballast.

\begin{tabular}{|c|c|c|}
\hline Reference Unit & Value & Reference \\
\hline $\begin{array}{l}\text { Passing vs. sieve size } \\
(\mathrm{mm}): 80-63-50-40- \\
31.5-22.4(\%)\end{array}$ & $\begin{array}{l}100-100-79.9- \\
30.6-1.2-0.3\end{array}$ & EN 933-1:2012 \\
\hline $\begin{array}{l}\text { Passing vs. sieve size } \\
(\mathrm{mm}): 0.063(\%)\end{array}$ & 0.5 & EN 933-1:2012 \\
\hline Voids index $K(\%)$ & 42 & Tosti et al. 2016 \\
\hline $\begin{array}{l}\text { Relative dielectric } \\
\text { permittivity ballast (-) }\end{array}$ & 6.5 & Tosti et al. 2016 \\
\hline $\begin{array}{l}\text { Relative dielectric } \\
\text { permittivity methacrylate } \\
(-)\end{array}$ & 4 & Tosti et al. 2016 \\
\hline $\begin{array}{l}\text { Relative dielectric } \\
\text { permittivity air (-) }\end{array}$ & 1 & - \\
\hline
\end{tabular}


In view of the assumptions made at Section 2.2., the 2D simulation domain in Figure 3 has been realized by imposing a voids/ballast grain ratio equal to $K$, and by assigning to each phase the relevant dielectric permittivity. The GPR antenna was simulated through a line source suspended in the air at the same height than the real case, emitting a pulse with the same central frequency. Finally, the GPR signal has been simulated using the FDTD technique run in the gprMax numerical simulator. The real signal has been compared with the synthetic one, after a standard post-processing aimed at increasing the signalto-noise ratio. The results from the comparison of these two signals are encouraging (Figure 4), as it is possible to recognize the well synchronised reflections from the interfaces between the different media (i.e., air-ballast and methacrylate-Perfect Electric Conductor (PEC) interfaces). The slight misalignment between the temporal positions of the signals at the methacrylate-PEC interface, is related to the particles configuration just below the line of source, which can actually affect and modify the total height of the sample.

\section{CONCLUSIONS}

This study focuses on the simulation of the Ground Penetrating Radar (GPR) signal carried out on a railway track bed. A typical scenario of ballast layer has been created by means of the Random Sequential Adsorption paradigm, whereas the synthetic signal has been reproduced using the Finite-Difference Time-Domain (FDTD) technique.

The simulation domain has been simplified into a two-dimensional (2D) scenario composed of circleshaped particles randomly sized and positioned, according to the grading of the aggregates used in the real GPR tests.

The air-solid ratio has been imposed to be consistent with that of the three-dimensional (3D) real-

\section{TRANSMITTER RECEIVER}

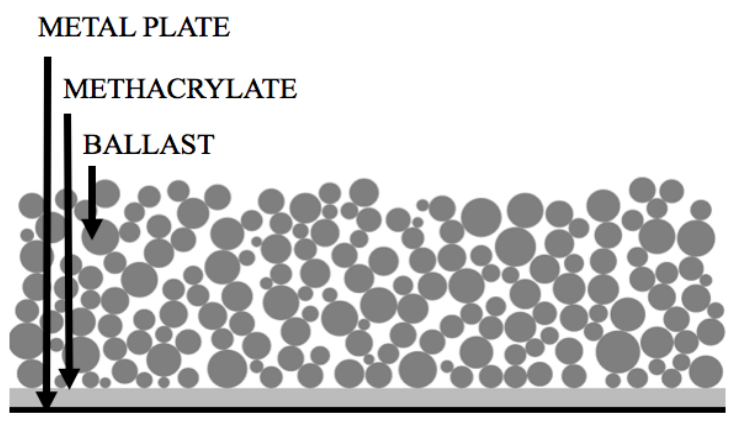

Figure 3. - The simulation domain.

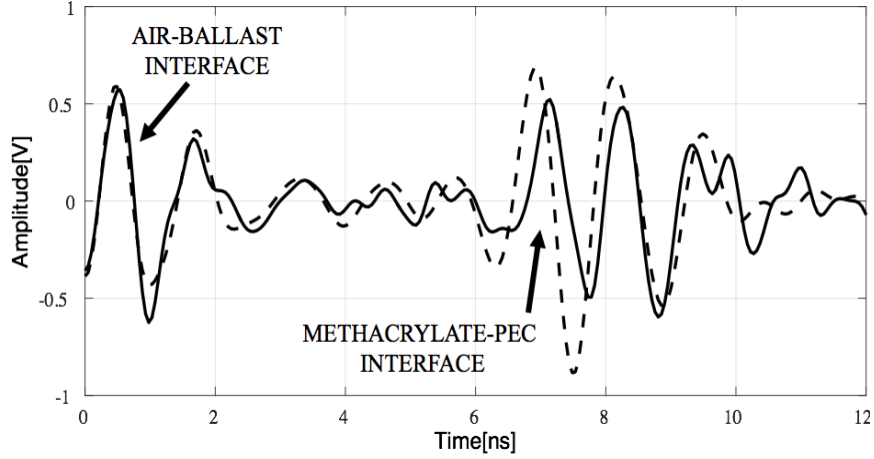

Figure 4. - Comparison between the real (solid) and the simulated (dashed) signals.

-life case, in order to ensure a dielectric behaviour representative of the real-case conditions. The methodology has been finally validated by comparing simulated and real GPR signals collected in the laboratory environment within a methacrylate tank. The promising outcomes highlight the potential of the method in simulating the electromagnetic response of a railway track bed.

Future perspectives for the application of this methodology might be focused on the characterisation of the ballast grading and the prediction of the ballast fouling.

\section{ACKNOWLEDGEMENTS}

The authors would like to express their gratitude to Mr Spartaco Cera, from Roma Tre University, for the unvaluable support during the laboratory activities. Special thanks to Clax Italia s.r.l. for manufacturing the methacrylate tank. This work has benefited of the network activities carried out within the EU-funded COST Action TU1208 "Civil Engineering Applications of Ground Penetrating Radar."

\section{REFERENCES}

Al-Qadi I.L., Xie W. \& Roberts R. 2008. "Time-Frequency Approach for Ground Penetrating Radar Data Analysis to Assess Railroad Ballast Condition, Research in Nondestructive Evaluation, 19, 219-237.

Al-Qadi I.L., Xie W., Roberts R. \& Leng Z. 2010. Data Analysis Techniques for GPR Used for Assessing Railroad Ballast in High Radio-Frequency Environment, Journal of Transportation Engineering, 136(4): 392-399.

Benedetto A. \& Pajewski L. , Eds. 2015. Civil Engineering Applications of Ground Penetrating Radar, Springer Book Series: "Springer Transactions in Civil and Environmental Engineering".

De Chiara F., Fontul S. \& Fortunato E. 2014. GPR Laboratory Test for Railways Materials Dielectric Propriety Assessment, Remote Sensing, 6, 9712-9728.

EN 933-1:2012 2012. Tests for geometrical properties of aggregates - Part 1: Determination of particle size distribution - Sieving method. European Committee for Standardization

Feder J. 1980. Random Sequential Adsorption, Journal of theoretical Biology, 87, 237-254 
Giannopoulos A. 2005. Modelling Ground Penetrating Radar by gprMax, Construction and Building Materials, 19,755-7.

Hugenschmidt, J. 2000. Railway track inspection using GPR, In Journal of Applied Geophysics, 43, pp. 147-155

Indraratna, B. 2016. 1st Ralph Proctor lecture of ISSMGE. Railroad performance with special reference to ballast and substructure characteristics, In Transportation Geotechnics, 7, pp. 74-114.

Indraratna B., Nimbalkar S. S., Ngo N. T. \& Neville T. 2016. Performance improvement of rail track substructure using artificial inclusions - Experimental and numerical studies, Transportation Geotechnics 8, 69-85A.

Lee J. W. 2000. Irreversible random sequential adsorption of mixtures, Colloids and Surfaces A: Physicochemical and Engineering Aspects, 165, 363-372.

Olhoeft G.R. \& Selig E.T. 2002. Ground penetrating radar evaluation of railroad track substructure conditions, 9th International Conference on Ground Penetrating Radar, Santa Barbara, USA.

Pirrone D. \& Pajewski L. 2015. E2GPR - Edit your geometry, Execute GprMax2D and Plot the Results!, Proc. 2015 IEEE $15^{\text {th }}$ Mediterranean Microwave Symposium, 30 November2 December 2015, Lecce, Italy, pp. 1-4.

Roberts R., I. Al-Qadi I.L., Tutumluer E., Boyle J. and T.R. Sussmann 2006. Advances in Railroad Ballast Evaluation Using 2 GHz Horn Antennas", in Proc. 11th International Conference on Ground Penetrating Radar, June 19-22.
Roberts R., Schutz A., Al-Qadi I.L. \& Tutumluer E. 2007. Characterizing railroad ballast using GPR: recent experiences in the United States, in Proc. of the 2007 4th International Workshop on Advanced Ground Penetrating Radar (IWAGPR 2007), Naples, Italy.

Selig E. T. \& Waters J. M. 1994. Track geotechnology and substructure management. In Thomas Telford ed., London.

Sharif A., Harkness J., Le Pen L., Powrie W. \& Zervos A. 2016. Numerical modelling of railway ballast at the particle scale, International Journal for Numerical and Analytical Methods in Geomechanics, vol. 40, issue 5, pp. 713-737.

Talbot J., Tarjus G. \& Van Tassel P.R. \& Viot P. 2000. From car parking to protein adsorption: an overview of sequential adsorption processes, Colloids and Surfaces A: Physicochemical and Engineering Aspects 165, 287-324.

Tosti F., Benedetto, A. Bianchini Ciampoli, L. \& Calvi, A., 2016. Laboratory Investigations for the Electromagnetic Characterization of Railway Ballast through GPR, in: Proc. of 16th International Conference of Ground Penetrating Radar (GPR 2016). doi: 10.1109/ICGPR.2016.7572605

Thakur P. K., Indraratna B. \& Vinod J. S. 2009. DEM simulation of effect of confining pressure on ballast behavior, In Hamza M., Shahien M. \& El-Mossallamy Y. (eds), 17th International Conference on Soil Mechanics and Geotechnical Engineering,602-605. 